\title{
Effets des barrages sur la continuité sédimentaire
}

\author{
Benoît CAMENEN ${ }^{1}$
}

${ }^{I}$ Irstea centre de Lyon Villeurbanne, UR HHLY, 5 rue de la Doua, Villeurbanne-e-mail: benoit.camenen@irstea;fr

\begin{abstract}
RÉSUMÉ. - Ce travail présente au travers de quelques exemples français les effets des barrages sur le transport solide et donc sur l'envasement des rivières en distinguant tout particulièrement les trois principaux modes de transport : le charriage, la suspension graduée et la suspension de lessivage. Une discussion sur le terme de transparence aux flux sédimentaire est proposée selon le type de barrage, sur ses possibles définitions et leur interprétation. Le charriage est discuté principalement au travers des ouvrages au fils de l'eau sur l'Arc-en-Maurienne, la suspension graduée avec la série d'ouvrages de la Basse-Isère, et la suspension de lessivage avec le Barrage de Génissiat sur le Haut-Rhône français.
\end{abstract}

Mots-clés : transport solide, barrage, continuité sédimentaire, transparence sédimentaire

\section{Effects of dams on sediment continuity}

\begin{abstract}
A discussion on the effects of dams on sediment transport and deposition is presented in this paper through some French examples: River dams on the Arc River, on the Isère River, and on the French Upper-Rhône River. In particular, a clear distinction is made between the three main modes of sediment transport, i.e. bedload, graded suspended load, and washload. Dam impacts on sediment fluxes and more especially the terminology sediment "transparency" or continuity is also discussed.
\end{abstract}

Key-words: sediment transport, dam, sediment continuity

\section{INTRODUCTION}

L'effet des barrages sur les sédiments est indirect, car il résulte d'une modification des conditions hydrauliques $\mathrm{du}$ fait de la présence d'une retenue en amont du barrage et de la modification des caractéristiques hydrologiques à l'aval du barrage. Les retenues de barrage sont caractérisées par une augmentation artificielle de la profondeur d'eau de l'amont vers l'aval et donc d'une réduction des contraintes hydrauliques. Un découpage longitudinal des retenues allongées en trois zones est proposé par Thornton et al. [1981] : un tronçon fluvial situé à l'amont de la retenue et peu influencé par la retenue ; une retenue amont située dans la zone influencée par la retenue la plus en amont avec un dépôt en delta des sédiments les plus grossiers (graviers puis sables); et enfin une retenue aval proche du barrage et plus profonde favorisant un dépôt des sédiments les plus fins [Fan et Morris, 1992a]. Un tel système implique donc un tri granulométrique d'amont en aval qui a des conséquences non-négligeables sur la gestion des barrages [Fan et Morris, 1992b]. L'impact en aval des barrages est tout d'abord lié à la réduction des débits et de la distribution des crues qui va donc impacter la capacité de transport de la rivière [Brandt, 2000] mais surtout au déficit sédimentaire induit par la présence de l'ouvrage transversal [Kondolf, 1997].

Aujourd'hui, la Directive Cadre Européenne (DCE) rend obligatoire le maintien d'un transport suffisant des sédiments en aval d'ouvrage transversaux sur certains cours d'eau (Article L.214-17 du CE). De plus, la préservation du bon état écologique implique un maintien du substrat alluvial. Cette continuité sédimentaire au travers d'un ouvrage transversal peut-être caractérisée par le coefficient de continuité sédimentaire qui correspond au rapport $\alpha_{C S}$ entre le transport solide entrant $Q_{s, \text { in }}$ et le transport solide sortant $Q_{s, \text { out }}$ :

$$
\alpha_{C S}=\frac{Q_{s, \text { in }}}{Q_{s, \text { out }}},
$$

Une telle définition mérite cependant d'être précisée sur l'aspect temporel (entre un flux instantané et un flux moyen annuel) mais aussi en termes de qualité (conservation de la granulométrie du sédiment). En conséquence, il est fondamental de bien distinguer les trois principaux modes de transport en lien avec les classes de sédiments transportées, soit le charriage (sables, graviers et galets), la suspension graduée (sables fins) et la suspension de lessivage (argile, limons). Pour le charriage et la suspension graduée, une estimation des flux peut être réalisée expérimentalement grâce à une mesure directe (préleveurs), indirecte instantanée (géophones, hydrophones, ADCP, etc.) ou indirecte moyennée (bilans bathymétriques, chaînes d'érosion). Elle rester néanmoins difficile et coûteuse. En supposant un lit en équilibre avec des apports solides suffisants, il est aussi possible d'estimer les flux par charriage et suspension graduée à partir de conditions hydrauliques locales à l'aide de formules semi-empiriques. A l'opposé, la suspension de lessivage se mesure relativement aisément en faisant l'hypothèse d'une homogénéité des concentrations sur la section de mesure en associant un turbidimètre calé avec un préleveur automatique à une station de mesure des débits (ensemble appelé communément station hydro-sédimentaire) :

$$
Q_{s, s l}(t)=Q(t) \times C(t)
$$

où $Q_{s, s l}(t)$ est le débit solide instantané transporté en suspension de lessivage, $Q(t)$ le débit liquide et $C(t)$ la concentration en sédiments. La suspension de lessivage ne peut être modélisée à partir des seules conditions 
hydrauliques locales et nécessite une connaissance des apports de l'amont. Dans le langage courant, on associe souvent le terme MES (Matériau En Suspension) à la suspension de lessivage alors que cela inclut aussi la suspension graduée de sable.

L'objectif de cet article est donc de discuter l'impact de différents barrages sur la continuité sédimentaire en distinguant tout particulièrement les trois modes de transport présentés précédemment.

\section{EFFETS DES BARRAGES SUR LA CONTINUITÉ SÉDIMENTAIRE}

\section{II.1. Barrage au fil de l'eau de faible capacité : exemple de St-Martin-la-Porte sur l'Arc}

Le barrage de St-Martin-la-Porte (SMLP) est situé sur l'Arc-en-Maurienne en amont de St-Jean-de-Maurienne. Ce barrage au fil de l'eau a une très faible capacité de stockage et fait l'objet de forts dépôts de grossiers à la queue de la retenue et de fines proches du barrage (Figure 1). Une chasse de barrages est réalisée chaque année au mois de juin sauf si une crue supérieure à la crue annuelle est observée (pour laquelle le barrage est totalement ouvert). Ces chasses sont efficaces pour évacuer les sédiments fins accumulés lors de l'année précédente, elles permettent aussi un transport important de matériaux grossiers mais a priori insuffisant par

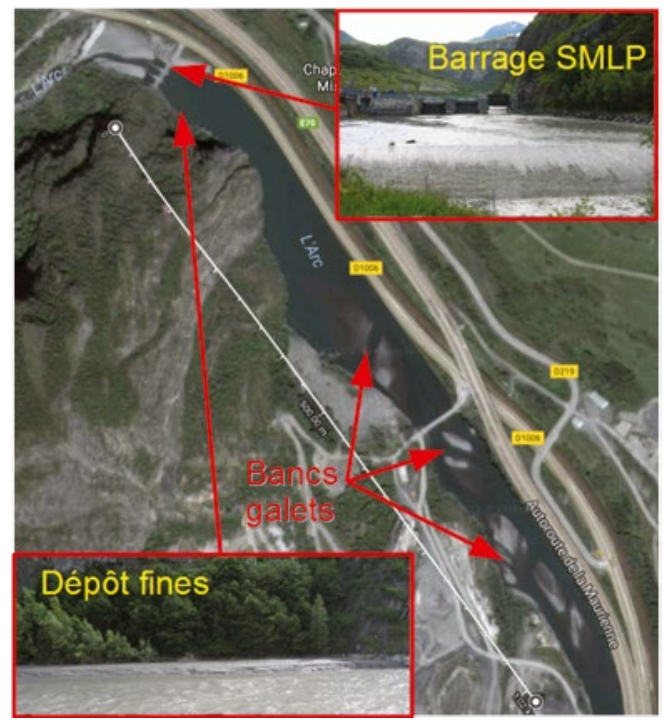

Figure 1: Photographie aérienne de la retenue de St-Martin-la-Porte. rapport aux apports de l'amont. Des mesures de charriage, $15 \mathrm{~km}$ en aval de SMLP pendant les chasses de 2011 et 2012, ont montré que le seuil de mise en mouvement des grossiers dans le chenal de la rivière était d'environ $50 \mathrm{~m}^{3} / \mathrm{s}$ et que la capacité de transport était a priori atteinte [Camenen et al., 2012]. Même si la rivière est globalement en faible incision en aval de SMPL, les apports restent suffisants pour maintenir un certain nombre de tronçons en équilibre dynamique [Jaballah et al., 2015]. Concernant le charriage, nous avons donc $\alpha_{C S, b}=0$ si le barrage est fermé et $\alpha_{C S, b} \approx 1$ lors des événements de chasse ou crue où le barrage est ouvert.

Aucune mesure de flux de sables n'a pu être réalisée sur ce site mais la part non-négligeable des sables dans la composition des bancs de galets indique un comportement a priori similaire aux graviers. Concernant la suspension de lessivage, le tableau 1 présente un bilan interannuel à la station hydro-sédimentaire de Pontamafrey située en aval de St-Jean-de-Maurienne avec la part du principal événement de l'année. On peut remarquer que la part des chasses au flux annuel est généralement de 2 à $10 \%$ (en 2016, la chasse a atteint un débit max de près de $200 \mathrm{~m}^{3} / \mathrm{s}$ au lieu de $Q_{\max }=120 \mathrm{~m}^{3} / \mathrm{s}$ habituellement et une crue de l'Arvan a eu lieu en même temps). A contrario, les crues comme celle de juin $2013\left(Q_{\max } \approx 350 \mathrm{~m}^{3} / \mathrm{s}\right)$ peuvent transporter une grande partie du flux annuel. Il est donc possible d'avoir un coefficient de continuité sédimentaire supérieur à 1 sur un événement particulier où la retenue est érodée mais sur le long terme, ce coefficient reste inférieur ou égale à 1 . Ceci implique qu'en régime normal (vannes de SMLP fermées), $\alpha_{C S, S l}<1$ (la majorité des fines passent, seule une fraction est interceptée par la retenue); lors de chasse, $\alpha_{C S, s l}>1$ (érosion de la retenue); et lors d'une crue majeure, $\alpha_{C S, s l}=1$.

\section{II.2. Barrage au fil de l'eau de grande capacité : exemple de la chaîne de barrage de la Basse-Isère}

La chaîne de barrages de la Basse-Isère est composée de cinq barrages (Beauvoir, St-Hilaire, Pizançon, La Vanelle, et Beaumont-Monteux) avec des retenues de volumes conséquents allant de $3,3 \times 10^{6} \mathrm{~m}^{3}$ à $13,5 \times 10^{6} \mathrm{~m}^{3}$. Cette chaîne de barrages est soumise à une sédimentation de matériaux fins assez importante nécessitant de réaliser des chasses de barrages. Il est aussi à noter que la retenue de St-Egrève située entre la ville de Grenoble et Beauvoir intercepte aussi une quantité importante de sédiments. Si le fond de l'Isère est principalement constitué de grossiers, les apports à la chaîne de barrages de la Basse-Isère sont aujourd'hui quasi nuls. Deux stations hydro-sédimentaires gérées par EDF et situées en amont (Tullins) et en aval (Beaumont-Monteux) de ce système nous indiquent que le transport moyen annuel en suspension de lessivage est d'environ $3 \times 10^{6} t$ et qu'environ

Tableau 1 : Bilan de flux de suspension de lessivage mesuré à la station de Pontamafrey (aval de St-Jean-de-Maurienne).

\begin{tabular}{|c|c|c|c|c|}
\hline Année & Flux annuel (tonnes) & Événement majeur & $\begin{array}{c}\text { Flux lié à l'événement } \\
\text { (tonnes) }\end{array}$ & $\%$ année \\
\hline 2011 & $6,5 \times 10^{5}$ & Chasse juin 2011 & $1,7 \times 10^{4}$ & 2,5 \\
\hline 2012 & $5,1 \times 10^{5}$ & Chasse juin 2012 & $4,9 \times 10^{4}$ & 9,6 \\
\hline 2013 & $12,8 \times 10^{5}$ & Crue juin 2013 & $54,0 \times 10^{4}$ & 42 \\
\hline 2014 & $5,3 \times 10^{5}$ & Chasse juin 2014 & $1,4 \times 10^{4}$ & 2,6 \\
\hline 2015 & $6,1 \times 10^{5}$ & Chasse juin 2015 & $2,1 \times 10^{4}$ & 2,8 \\
\hline 2016 & $3,9 \times 10^{5}$ & Chasse juin 2016 & $6,7 \times 10^{4}$ & 17 \\
\hline
\end{tabular}


$50 \%$ de ce flux se déposent dans les retenues (EDF-DPIH, 2015). Il est à noter cependant que ce chiffre dépend fortement des événements et surtout de la granulométrie des MES. En effet, une lave torrentielle dans le bassin versant de l'Arc peut générer un pic de concentration de sédiments très fins (argile) qui passe l'ensemble des ouvrages sans se déposer et peut ainsi être visible jusqu'au Rhône. Les chasses de la Basse-Isère sont réalisées en descente de crue de l'Isère pour des conditions de débit de l'Isère et du Rhône précises pouvant être résumées simplement à $Q_{\text {Isère }}>900 \mathrm{~m}^{3}$ et $Q_{\text {Rhône }}>1200 \mathrm{~m}^{3}$. Ainsi, un événement de chasse de la Basse-Isère peut se décomposer en trois périodes avec la phase de crue (durée de quelques jours où les vannes de fond des ouvrages sont ouvertes mais les retenues non effacées), la phase de chasse (durée d'une semaine correspondant au début d'effacement de la retenue de BeaumontMonteux à la remontée du barrage de St-Hilaire) et, enfin, la phase de remplissage (durée de deux semaine environ jusqu'à la remontée du barrage de Beaumont-Monteux). Lors de la chasse de mai 2015 un très fort dépôt de sables a été constaté sur la partie aval de l'Isère, celui-ci s'est ensuite déplacé vers le canal d'amenée du Rhône impliquant d'importantes difficultés pour la navigation [Naudet et al., 2016].

Un bilan sédimentaire a été réalisé sur l'ensemble de la Basse-Isère suite à l'événement de mai 2015 (Tableau 1). Les volumes érodés dans les retenues et déposés en aval ont été calculés à partir de mesures bathymétriques réalisées

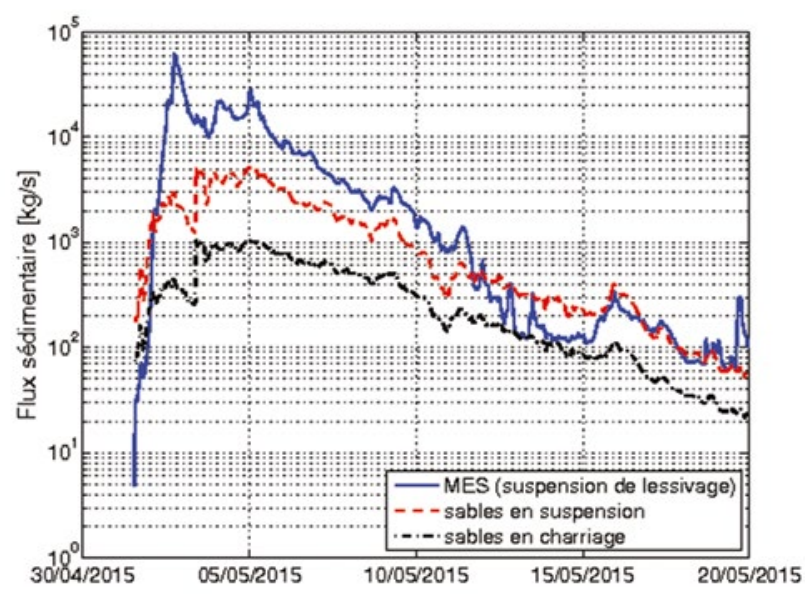

Figure 2 : Flux de sédiments en suspension de lessivage et charriage et suspension graduée de sables à la station de Beaumont-Monteux. avant et après l'événement et retranscris en masse à partir d'hypothèses de masse volumique des sédiments déposés. Les flux de suspension de lessivage amont et aval ont été calculés à partir des deux stations hydro-sédimentaires. Enfin, le flux de sable à Beaumont-Monteux a été estimé sur la base d'une courbe de tarage sédimentaire (cf. Camenen et al., 2012) calibrée à partir des dépôts de sables observés en aval. Un bilan sur la suspension de lessivage (stations hydro-sédimentaires) indique un apport des retenues de la Basse-Isère d'environ $5 \times 10^{6} \mathrm{t}$ d'argiles et limons auquel se rajoute un apport de $5 \times 10^{6} \mathrm{t}$ de sable correspondant au dépôt aval. Si le bilan est à peu près bouclé aux incertitudes près (masse érodée dans les retenue estimée à $7,5 \times 10^{6} \mathrm{t}$ au total), ces dernières restent cependant élevées avec sans doute une porosité surestimée, et donc un masse volumique sous-estimée dans les retenues.

La figure 2 présente les flux de suspension de lessivage mesurés à la station de Beaumont-Monteux ainsi que les flux charriés et en suspension graduée de sables calculés à partir de la courbe de tarage sédimentaire calées afin de pouvoir reproduire le volume de sable déposé sur la confluence avec l'Isère (diamètre médian du sable $d_{50}=0,2 \mathrm{~mm}$ ). Il apparaît donc que les flux de sables étaient d'un ordre de grandeur plus faible que la suspension de lessivage lors des phases de crue et de chasse mais du même ordre de grandeur lors de la phase de remplissage. Des mesures après l'événement ont, de plus, montré que ce flux de sable se tarissait très vite après la fermeture du barrage de Beaumont-Monteux (Naudet et al., 2016). On peut donc affirmer que pour le sable : $\alpha_{C S, s}=0$ en dehors des crues et des chasses et $\alpha_{C S, S}>1$ lors d'une crue-chasse.

\section{II.3. Grand barrage avec vanne de fond : exemple de Génissiat sur le Rhône}

Le barrage de Génissiat est le plus grand barrage du Haut-Rhône français avec une chute de $63 \mathrm{~m}$, une retenue de $23 \mathrm{~km}$ de long et un volume de $56 \times 10^{6} \mathrm{~m}^{3}$. L'ouvrage a pour originalité de disposer de trois orifices positionnés à différents niveaux : une vanne de fond, une vanne de demi-fond, et un évacuateur de surface. Ce système permet d'optimiser la gestion d'une chasse ou d'un accompagnement des chasses des barrages suisses en amont en maitrisant les concentrations en MES rejetées à l'aval [Peteuil et al, 2013]. La retenue de Génissiat a subi une forte sédimentation depuis sa construction en 1954 en particulier sur sa partie aval où près de $40 \mathrm{~m}$ de dépôt sont aujourd'hui observés (Figure 3a). La distribution granulométrique est, de plus, caractéristique d'une retenue allongée avec un tronçon fluvial pavé $\left(d_{50} \approx 100 \mathrm{~mm}\right)$, une retenue amont sableuse (

Tableau 2 : Bilan sédimentaire sur l'ensemble de la Basse-Isère suite à la crue-chasse de mai 2015.

\begin{tabular}{|c|c|c|c|c|}
\hline Site & Mesures / modèles & $\begin{array}{c}\text { Volumes } \\
\left(10^{6} \mathrm{~m}^{3}\right)\end{array}$ & $\begin{array}{c}\text { Masses } \\
\left(10^{6} \mathrm{t}\right)\end{array}$ & $\begin{array}{c}\text { Incertitudes } \\
\left(10^{6} \mathrm{t}\right)\end{array}$ \\
\hline Flux entrants amont & $\begin{array}{c}\text { Station hydro-sédimentaire } \\
\text { (Tullins) }\end{array}$ & & 2,5 & $0,5(20 \%)$ \\
\hline Barrages Basse-Isère & Evolution bathymétrique barrages & 4,8 & 7,5 & $2,5(30 \%)$ \\
\hline Flux sortants aval & $\begin{array}{c}\text { Station hydro-sédimentaire } \\
\text { (Beaumont-Monteux) }\end{array}$ & & 7,6 & $1,5(20 \%)$ \\
\hline & Modèle sable (Beaumont-Monteux) & & 4,8 & $1,0(20 \%)$ \\
\hline Dépôts aval & $\begin{array}{c}\text { Evolution bathymétrique } \\
\text { confluence Isère-Rhône }\end{array}$ & 3,3 & 5,3 & 1 \\
\hline
\end{tabular}


$\left.d_{50} \approx 0.5 \mathrm{~mm}\right)$, et une retenue aval limoneuse $\left(d_{50} \approx 50 \mu \mathrm{m}\right)$ (Figure 3b). Guertault et al. [2014] ont montré à partir d'une étude des bilans sédimentaires de la retenue que si le bilan variait fortement d'une chasse du Haut-Rhône à l'autre en fonction de la gestion de cet événement, les évolutions en période d'interchasse impliquent en général une érosion de la retenue amont et une sédimentation sur la retenue aval.

La mise en place de stations hydro-sédimentaires en amont et en aval de la retenue en 2015 a montré que la concentration en aval du barrage (Pyrimont) est très proche de la concentration en amont de la retenue (Pougny) en régime normal. Seuls les principaux pics de concentrations sont atténués entre Pougny et Pyrimont (Figure 4a). Ainsi, il semblerait qu'il existe, en fonction des contraintes hydrauliques dans la retenue, une concentration en matières en suspension limite au-delà de laquelle du dépôt a lieu. Il faut de plus prendre en compte la variabilité des débits sur chacun des ouvrages en fonction des périodes de production d'électricité et des débits entrants dans le système (Lac Léman et Arve). En moyenne, il a été estimé en régime normal un taux de dépôt relativement faible (environ 20\%) par rapport aux flux transitant, soit $\alpha_{C S, s l} \approx 0.8$ [Guertault, 2015].

Concernant les grossiers, des mesures en période d'interchasse ont montré qu'il n'y avait aucun flux entrant lorsque la retenue amont (Chancy-Pougny) était fermée. Par contre, des mesures réalisées pendant les chasses de 2012 et 2016 ont montré un fort apport en sable, voire même un transport de graviers et de galets sur le tronçon fluvial mais la capacité de transport est rarement atteinte du fait des apports insuffisants [Guertault, 2015]. A l'aval du barrage de Génissiat, il a été observé un flux résiduel de sable lors des chasses uniquement. Il est à noter cependant que ces flux charriés restent négligeables par rapport aux flux de suspension de lessivage.

\section{CONCLUSIONS}

L'impact de différents barrages sur les flux sédimentaires a été abordé dans cet article en distinguant tout particulièrement les modes de transport et, par conséquent, la granulométrie des sédiments transportés. Il est bien évident qu'un barrage, quel qu'il soit, n'est pas « transparent » et a un impact plus ou moins élevé sur l'hydrologie (au moins sur les débits moyens à faibles, voire sur les crues si le barrage a la capacité et la fonction d'écrêter les crues) et donc sur le transport solide, en particulier pour les sédiments transportés en charge de fond. Les impacts sont bien sûr plus ou moins importants selon le type de barrage, sa taille et sa gestion. Lorsqu'un barrage est fermé, il capture en général la totalité des sédiments grossiers (charriage et suspension graduée) et une partie de la suspension de lessivage. Lorsque le barrage est ouvert, selon la gestion du barrage, il peut y avoir érosion de la retenue et ainsi un coefficient de continuité sédimentaire supérieur à 1 .

Le tableau 3 est proposé pour résumer les impacts des trois types de barrage discutés dans ce papier. On peut ainsi observer sur le long terme que l'impact d'un barrage de faible capacité reste insignifiant pour la suspension de

(a)
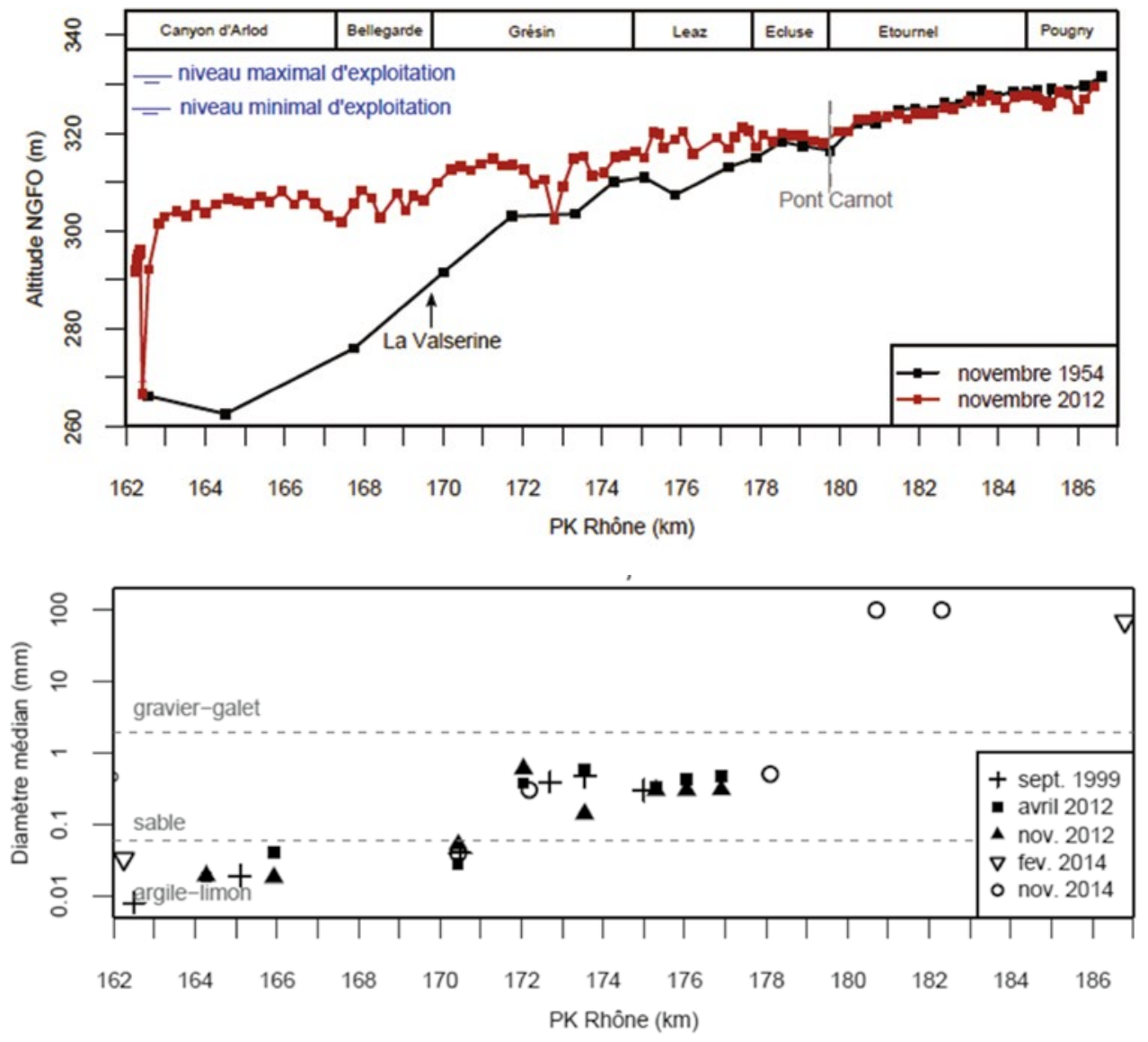

Figure 3 : Évolution bathymétrique de la retenue de Génissiat (a) et répartition spatiale de la granulométrie récente (b) (Guertault, 2015). 
(a)

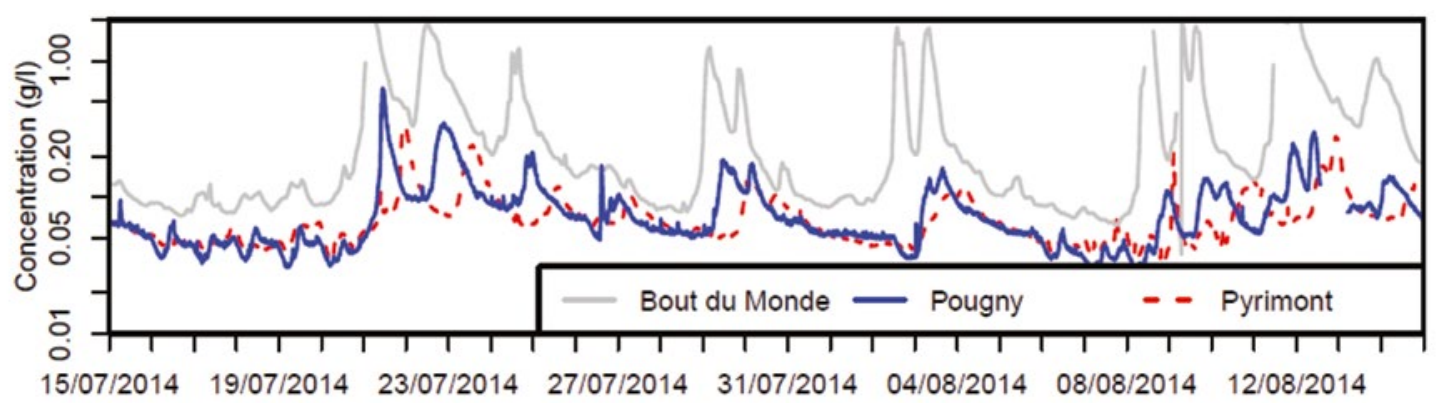

(b)

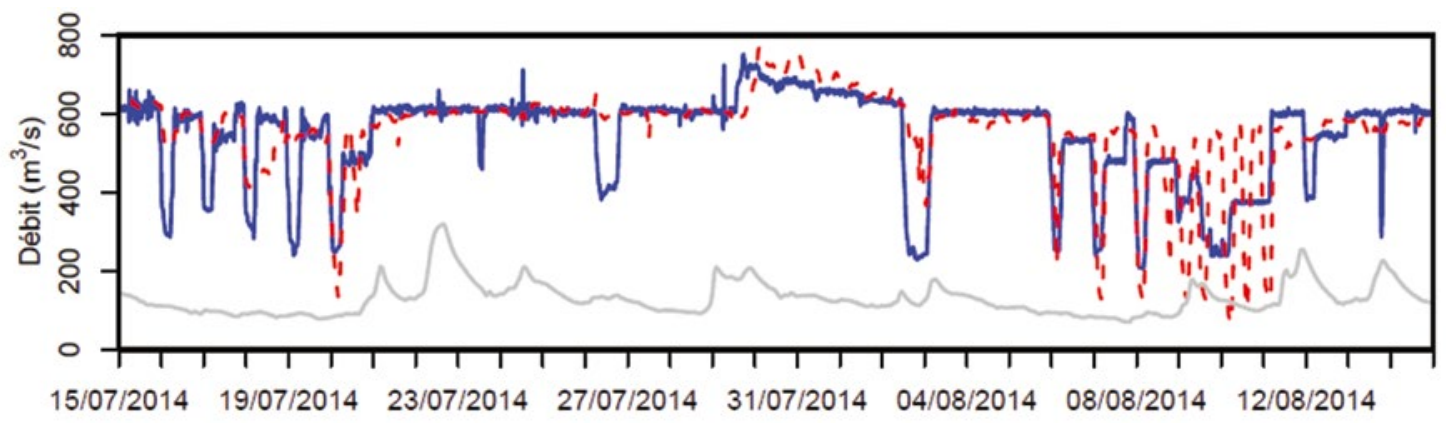

Figure 4 : Chronique de concentration (a) et de débit (b) sur l'Arve (station du Bout du Monde) et le Rhône en amont (Pougny) et en aval (Pyrimont) de la retenue de Génissiat (Guertault, 2015).

Tableau 3 : Estimation de la continuité sédimentaire selon le type de barrage et sa gestion en chasse/crue.

\begin{tabular}{|c|c|c|c|c|}
\hline Gestion & Type barrage & $\begin{array}{l}\text { Barrage au fil de l'eau } \\
\text { de faible capacité } \\
\text { (ex : St-Martin-la-Porte) }\end{array}$ & $\begin{array}{l}\text { Barrage au fil de l'eau } \\
\text { de grande capacité } \\
\text { (ex : Basse-Isère) }\end{array}$ & $\begin{array}{l}\text { Grand barrage avec } \\
\text { vannes de fond } \\
\text { (ex : Génissiat) }\end{array}$ \\
\hline \multirow[t]{3}{*}{ Période normale } & Charriage & $\alpha_{C S, b}=0$ & $\alpha_{C S, b}=0$ & $\alpha_{C S, b}=0$ \\
\hline & Suspension graduée & $\alpha_{C S, s} \approx 0$ & $\alpha_{C S, s}=0$ & $\alpha_{C S, s}=0$ \\
\hline & Suspension de lessivage & $0.9<\alpha_{C S, s l} \leq 1$ & $0.8<\alpha_{C S, s l} \leq 0,95 *$ & $0.7<\alpha_{C S, s l} \leq 0,9$ \\
\hline \multirow{3}{*}{$\begin{array}{l}\text { Période de } \\
\text { chasse ou/et } \\
\text { crue }\end{array}$} & Charriage & $\alpha_{C S, b}<1$ & $\alpha_{C S, b} \approx 0$ & $\alpha_{C S, b} \approx 0$ \\
\hline & Suspension graduée & $\alpha_{C S, S} \approx 1$ & $\alpha_{C S, s}>1$ & $\alpha_{C S, s}>0$ \\
\hline & Suspension de lessivage & $\begin{array}{c}\alpha_{C S, s l}>1 \text { (chasse) } \\
\alpha_{C S, s l} \approx 1 \text { (crue) }\end{array}$ & $\alpha_{C S, s l}>1$ & $\begin{array}{c}0.5<\alpha_{C S, s l}<2 \\
\text { (selon la gestion) }\end{array}$ \\
\hline \multirow{3}{*}{$\begin{array}{c}\text { Total } \\
\text { (moyenne } \\
\text { interannuelle) }\end{array}$} & Charriage & $\alpha_{C S, b}<1$ & $\alpha_{C S, b} \approx 0$ & $\alpha_{C S, b}=0$ \\
\hline & Suspension graduée & $\alpha_{C S, s} \approx 1$ & $\alpha_{C S, s}<1$ & $\alpha_{C S, s}>0$ \\
\hline & Suspension de lessivage & $\alpha_{C S, s l}=1$ & $\alpha_{C S, s l} \approx 1$ & $\alpha_{C S, s l}<1$ \\
\hline
\end{tabular}

lessivage et la suspension graduée de sable, et modéré pour le charriage. Pour un barrage au fil de l'eau de grande capacité, l'impact devient important pour le charriage mais toujours faible pour la suspension de lessivage, voire même pour la suspension graduée de sable. Enfin, pour un grand barrage avec vanne de fond, l'impact est alors très important pour le charriage et la suspension graduée de sable, mais modéré pour la suspension de lessivage.

Si le développement du réseau de stations hydro-sédimentaires actuel permet une meilleure estimation des impacts des ouvrages transversaux sur le flux de suspension de lessivage, il manque encore beaucoup de données pour estimer les impacts sur les sédiments plus grossiers lorsqu'il s'avère qu'il y a une continuité sédimentaire partielle. Il apparaît donc très important d'investir dans la mesure du charriage et de la suspension graduée pour mieux appréhender leur flux, en particulier en période de crue et/ou de chasse des barrages.

\section{RÉFÉRENCES}

BRANDT, S. A. (2000) - Classification of geomorphological effects downstream of dams. Catena, 240, 375-401.

Camenen, B., Jaballah, M., Geay, T., Belleudy, P., Laronne, J.B., \& LASKOWSKI, J.P. (2012) - Tentative measurements of bedload transport in an energetic alpine gravel bed river. River Flow, San Jose, Costa Rica, 379-386.

EDF-DPIH (2015) - Synthèse du suivi environnemental de la chasse de la basse-Isère du 3 au 26 mai 2015. Rapport technique, 72 p. 
FAN, J. ET MORRIS, G. L. (1992A) - Reservoir sedimentation. I : Delta and density current deposits. Journal of Hydraulic Engineering, 118(3), 354-369.

FAN, J. ET MORRIS, G. L. (1992B) - Reservoir sedimentation. II : reservoir desiltation and long term storage capacity. Journal of Hydraulic Engineering, 118(3), 370-384.

Guertault, L., Camenen B., Peteuil, C., Paquier, A. (2014) Long term evolution of a dam reservoir subjected to regular flushing events. Advances in Geosciences, 39, 89-94.

Guertault, L. (2015) - Évaluation des processus hydro-sédimentaires d'une retenue de forme allongée: application à la retenue de Génissiat sur le Haut-Rhône. Thèse de doctorat. Université Claude Bernard, Lyon 1, 262 p.

Jaballah, M., Camenen, B. \& Paquier, A (2015) - Alternate bar development in an alpine river following engineering works. Advance in Water Res, 81, 103-113.
Naudet, G., Dugué, V., Camenen, B, Le Coz, J., Dramais, G. \& PAquier, A. (2016) - Étude de la dynamique hydrosédimentaire au niveau de la confluence entre l'Isère et le Rhône. Rapport technique, $112 \mathrm{p}$.

Peteuil, C., Fruchard, F., Abadie, F., Reynaud, S., Camenen, B., \& GuERTAULT, L. (2013) - Sustainable management of sediment fluxes in reservoir by environmental friendly flushing: the case study of Genissiat dam on upper Rhône River (France). Proc. International Symposium on River Sedimentation, Kyoto, Japon, Sept. 2013.

KondOLF, G. M. (1997) - Hungry water: effects of dams and gravel mining on river channels. Environmental management, 21(4), 533-551.

Thornton, K. W., Kennedy, R. H., Carroll, J. H., Walker, W. W., GunKel, R. C. ET AshBy, S. (1981) - Reservoir sedimentation and water quality - an heuristic model. Proceedings of the symposium on surface water impoundments ", 2-5 Juin 1980, Minneapolis, Minesota, États-Unis, ASCE, New York, NY., 1, 654-661. 